\title{
Local Orientation Field Based Nonlocal Means Method for Fingerprint Image De-Noising
}

\author{
J. Zou, J. B. Feng, X. M. Zhang, M. Y. Ding
}

School of Life Science and Technology, Huazhong University of Science and Technology, Wuhan, China.

Email: xmboshi.zhang@gmail.com

Received May, 2013.

\begin{abstract}
The de-noising of the fingerprint image is one of the key tasks before the extraction of the minutiae in automatic fingerprint matching. When used for de-noising the fingerprint image, the nonlocal means method can not preserve the local minutiae in the fingerprint image very well. To address this problem, we propose a local orientation field based nonlocal means (NLM-LOF) method in this paper. Experimental results on the simulated and real images show that the proposed method can suppress noise effectively while preserving edges and details in the fingerprint image and it outperforms the state-of-art nonlocal means method in terms of qualitative metrics and visual comparisons.
\end{abstract}

Keywords: Fingerprint Image Denoising; Nonlocal Means Filtering; Orientation Field

\section{Introduction}

As one of the most important biometric technologies, fingerprint identification has been widely used in identity recognition. Usually, fingerprint identification relies heavily on the performance of the minutiae extraction algorithm[1]. However, due to complex identify conditio- ns, the acquired fingerprint images are usually contaminated with noise which is disadvantageous for the minutiae extraction. So it is desirable and crucial to design a robust filter to preserve the local minutiae and improve the clarity of the ridge structures.

Many de-noising algorithms have already been presented to remove noise in the fingerprint images. Liang, et.al [2] proposed the morphological amoebas method to simultaneously reduce noise and preserve useful details with the help of pilot images from canny edge detection. Liang, et.al [3] developed a combinatorial linear time algorithm to eliminate impulsive noise and useless components from fingerprint images using Euclidean distance transform. In [4], Bayesian de-noising in the wavelet domain was presented to realize fingerprint image de-noising. All these methods tend to damage edges and details in the fingerprint images because they only use local information in the images.

Different from the above mentioned methods, the non-local means filter recently proposed by Buades [5] takes advantage of the redundancy of similar patches in the images and estimates the considered pixel with a weighted average of all the pixels in its neighborhood or the whole image. However, the performance of the tradi- tional non-local means (TNLM) method will be greatly influenced by similarity window and similarity computation method. Many novel solutions have been proposed to address this problem such as the NLM-

Reprojections (NLM-R) method [6], the NLM using Shape Adaptive Patches (NLM-SAP) [7]. Although these improved methods perform better than the traditional NLM, they cannot preserve fringes and minutiaes effectively. In this paper, we propose a novel nonlocal means filter based on the estimation of the orientation field. Compared with above state-of-art de-noising methods, the proposed method is more robust and it can preserve minutiae better while suppressing noise in the fingerprint images.

\section{Our Method}

In the TNLM method, for the considered pixel $(m, n)$ in the noisy image, the corresponding non-local means de-noised intensity $\operatorname{NL}(m, n)$ in the search window $\Omega$ is calculated as [5]:

$$
N L(m, n)=\sum_{(p, q) \in \Omega} \frac{w(m, n, p, q) v(p, q)}{\sum_{(p, q) \in \Omega} w(m, n, p, q)}
$$

where $v(p, q)$ denotes the intensity of pixel $(p, q)$, $w(m, n, p, q)$ denotes the similarity of two pixels $(m, n)$ and $(p, q)$ and it is computed as:

$$
w(m, n, p, q)=e^{-\frac{\left\|v_{s}(m, n)-v_{s}(p . q)\right\|_{2, a}}{h_{1}{ }^{2}}}
$$


where $h_{1}$ means the decay parameter. $v_{s}(m, n)$ and $v_{s}(p, q)$ denote the intensities of similarity windows $s$ centered at $(m, n)$ and $(p, q) \cdot \|_{2, a}$ denotes the Gaussian weighted Euclidean distance convolved with a Gaussian kernel of standard deviation $a$. As we can see, the TNLM method utilizes the geometrical configuration in a neighbourhood to determine the pixel similarity, but its ignorance of the orientation field will degrade its performance in restoring the fingerprint image. Considering that a well estimated orientation field can facilitate representing geometrical features effectively, we introduce the orientation field into the traditional nonlocal means to obtain improved de-noised results. The local orientation of each block centered at pixel $(m, n)$ is estimated as [1]:

$$
\begin{aligned}
& V_{X}(m, n)=\sum_{u=m-\frac{W}{2}}^{m+\frac{W}{2}} \sum_{v=n-\frac{W}{2}}^{n+\frac{W}{2}} 2 \partial_{X}(u, v) \partial_{y}(u, v) \\
& V_{y}(m, n)=\sum_{u=m-\frac{W}{2}}^{m+\frac{W}{2}} \sum_{v=n-\frac{W}{2}}^{n+\frac{W}{2}}\left(\frac{2}{\partial_{x}}(u, v)-\frac{2}{\partial y}(u, v)\right) \\
& \Theta(m, n)=\frac{1}{2} \tan ^{-1}\left(\frac{V_{y}(m, n)}{V_{x}(m, n)}\right)
\end{aligned}
$$

where $V_{y}(m, n)$ and $V_{x}(m, n)$ denote the gradients of pixel $(m, n)$,which is calculated by Sobel operator[8]. $\Theta(m, n)$ is the least square estimate of the local ridge orientation of the block $W$ centered at pixel $(m, n)$. Then we adjust the weigh $\{w(m, n, p, q)\}_{(p, q) \in \Omega}$ using orientation information $\Theta$.

$$
w(m, n, p, q)=e^{-\frac{\left\|v_{s}(m, n)-v_{s}(p . q)\right\|_{2, a}}{h_{1}{ }^{2}}} e^{-\frac{|\Theta(m, n)-\Theta(p, q)|^{2}}{h_{2}{ }^{2}}}
$$

where $h_{2}$ is the decay parameter. It can be seen from (6) that different from the TNLM method, the proposed method determines the weight by utilizing both the gray values of image patches and the local orientation field.

To demonstrate the superiority of the proposed method in weight calculation, we use the noisy images with standard deviation $\sigma \in\{50,60,70\}$ shown in Figure 1(a) and compute the distribution of the similarity between the center pixel and other pixels in each test image. Figure 1(b) and Figure 1(c) shows the weight distribution for the two compared methods, where its values go from one (white) to zero (black). It can be seen from Figure 1 that our method can determine the similarity more effectively than the TNLM method, especially at high noise corruption.

\section{Experiments}

In this section, the fingerprint image simulated using fingerprint generator software released at http://www.ia.c as.cn/kygz/kycg/rjdj/200910/t20091010_2542737.html and the real fingerprint image are used as the test images. For simulation experiments, gaussian white noise with the standard deviation $\sigma \in\{20,30,40,50\}$ is added to the simulated fingerprint image. The restoration performance of the TNLM, the NLM-R, the NLM-SAP and the proposed NLM-LOF is appreciated using peak signal-tonoise ratio (PSNR) and structural similarity
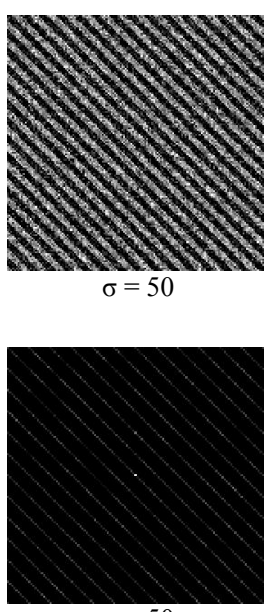

$\sigma=50$

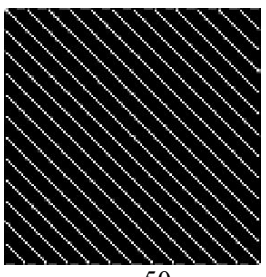

$\sigma=50$

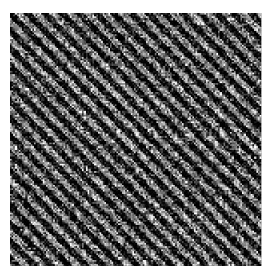

$\sigma=60$

(a)

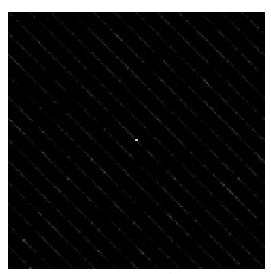

(b)

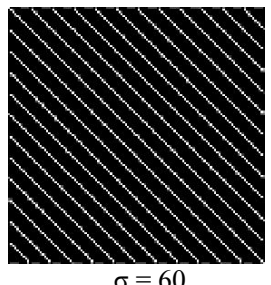

(c)

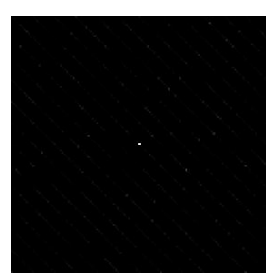

$\sigma=70$

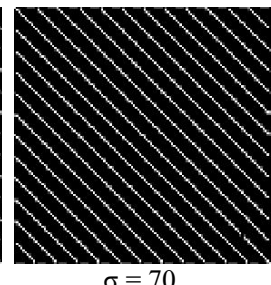

$\sigma=70$

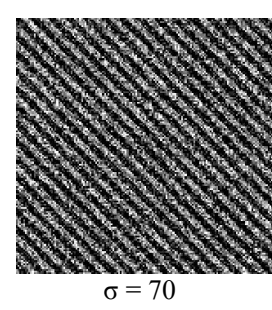

Figure 1. The distribution of weight similarity between the center pixel and other pixels in the noisy images. (a). noisy images with different standard deviations, (b). the weight distribution for the TNLM method, (c). the weight distribution for the NLM-LOF.

index [8] (SSIM). In all the experiments, we choose the search window size and the similarity window size to be $5 \times 5$ and $3 \times 3$, respectively. The filtering parameter $h$ (the same for h1 in the NLM-LOF) is fixed to $6^{*} \sigma$ and $h_{2}$ is tuned to obtain the best restoration performance for the proposed NLM-LOF. Table 1 lists the PSNR and SSIM values of all the de-noising methods operating on the corrupted fingerprint image. The observation from Table 1 demonstrates that our method has the best restoration performance in that it outperforms the compared methods in terms of the PSNR and SSIM measurement.

To visualize such improvement, we illustrate the de-noised images in Figure 2. As we can see, Figure 2(d) is more similar to the ground truth than Figure 2(c) and the edges are significantly protected while the TNLM (Figure 2(c)) oversmooth the whole image. 
Besides, we test the two methods on a real fingerprint image shown in Figure 3(a). Figure 3(b) is the zoomed view of labeled region In Figure 3(a). Much noise still exists in the image de-noised by the TNLM method (Figure 3(b)) and the important anatomical details are blurred. By comparison, the NLM-LOF can suppress noise effective- ly while preserving the anatomical details very well as shown in Figure 3(d).

\section{Conclusions}

We have proposed the local orientation field based nonlocal means method. Compared with the state-of-art nonlocal means filter, the proposed method can de-noise the fingerprint images more effectively because it utilizes more feature information to represent the structural similarity of pixels in the image. Experiments on simulated and real fingerprint images demonstrate the effectiveness of the proposed method in restoring the corrupted fingerprint image in terms of noise reduction and detail preservation.

Table 1. Comparisons of the PSNR values and SSIM valuesof the de-noised results on different standards noisy images.

\begin{tabular}{ccccc}
\hline \hline & \multicolumn{4}{c}{ PSNR/SSIM } \\
\cline { 2 - 5 }$\sigma$ & TNLM & NLM-R & NLM-SAP & NLM-LOF \\
\hline \multirow{2}{*}{20} & $24.77 / 0.9535$ & $26.72 / 0.9747$ & $26.56 / 0.9748$ & $29.19 / 0.9773$ \\
30 & $21.76 / 0.9061$ & $22.75 / 0.9308$ & $23.56 / 0.9467$ & $25.78 / 0.9601$ \\
40 & $19.50 / 0.8393$ & $19.97 / 0.8571$ & $21.28 / 0.9038$ & $23.73 / 0.9313$ \\
50 & $17.81 / 0.7623$ & $17.92 / 0.7579$ & $19.27 / 0.8354$ & $20.28 / 0.9066$ \\
\hline \hline
\end{tabular}

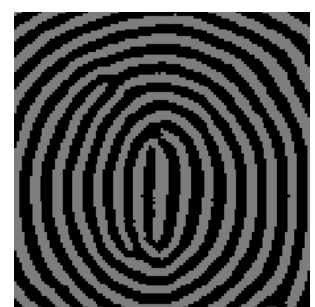

(a)

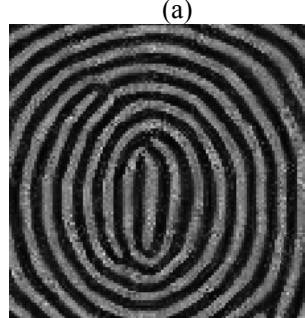

(c)

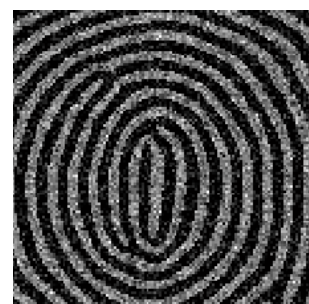

(b)

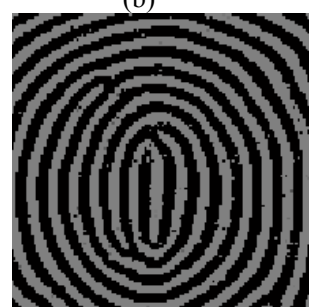

(d)
Figure 2. De-noising experiment on a simulated image. a. the original image, b. noisy image $(\sigma=40)$, c. result with NLM, d. result with NLM-LOF.

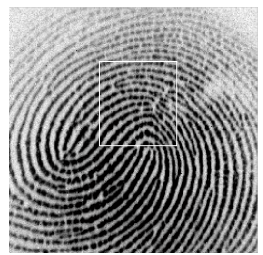

(a)

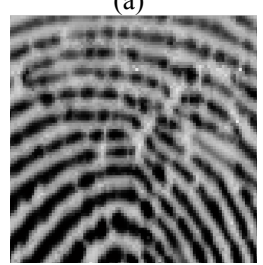

(c)

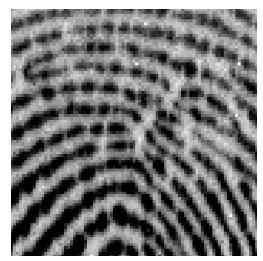

(b)

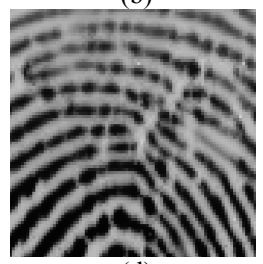

(d)
Figure 3. Comparisons of the de-noising results for the TNLM and NLM-LOF operating on a real fingerprint image. a.original image, $b$. zoomed view of the labeled region, c. result with TNLM, d.result with NLM-LOF.

\section{Acknowledgements}

This work was partly supported by the National Natural Science Foundation of China (Grant No.:30911120497), the National 973 project (Grant No.: 2011CB933103), the Project of the National 12th-Five Year Research Program of China (Grant No.: 2012BAI13B02) and Project of National High Technology Research and Development Program of China (Grant No.:2013AA040206).

\section{REFERENCES}

[1] L. Hong, Y. F. Wan and A. Jain, "Fingerprint Image Enhancement Algorithm and Performance Evaluation," IEEE Transactions on Pattern Analysis and Machine Intelligence, Vol. 20, No. 8, August 1998.

[2] Y. Y. Liang and P. Z. Wen, "Fingerprint Image Denoising using Morphological Amoebas," International Conferences on Intelligent Computing and Integrated Systems (ICISS), 2010, pp.197 - 200

[3] X. F. Liang and T. ASANO, "A Linear Time Algorithm for Binary Fingerprint Image Denoising Using Distance Transform," IEICE TRANS. INF. \& SYST., Vol. E89-D, No.4, April 2006.

[4] Portilla, J. Strela, V. Wainwright and M. J. Simoncelli, "Image Denoising using Scale Mixtures of Gaussians in the Wavelet Domain," IEEE Transactions on Image Processing, Vol. 12, No. 11, 2003, pp. 1338-1351. doi:10.1109/TIP.2003.818640

[5] A. Buades, B. Coll and J. Morel, "A Nonlocal Algorithm for Image Denoising," IEEE International Conferences on Computer Vision and Pattern Recognition (CVPR), Vol. 2, 2005, pp. 60-65.

[6] J. Salmon and Y. Strozecki, "From Patches to Pixels in Non-Local methods:Weighted-Average Rep-rojection," International Conferences On Image Processing (ICIP), 2010, pp. 1929-1932.

[7] C.-A. Deledalle J. Salmon and V. Duval, "Non-Local 
Methods with Shape-Adaptive Patches (NLM- SAP)," Journal of Mathematical Imaging and Vision (JMIV), Vol. 43, 2012, pp. 103-120. doi:10.1007/s10851-011-0294-y

[8] Z. Wang, A. C. Bovik, H. R. Sheik and E. P. Simoncelli,
"Image Quality Assessment: From Error Visibility to Structural Similarity," IEEE Transactions on Image Processing, Vol. 13, No. 4, Apr. 2004, pp. 600-602. doi:10.1109/TIP.2003.819861 